\title{
Calorimetric and acoustic emission study of martensitic transformation in single-crystalline $\mathrm{Ni}_{2} \mathrm{MnGa}$ alloys
}

\author{
László Z. Tóth, Sándor Szabó, ${ }^{*}$ Lajos Daróczi, and Dezső L. Beke \\ Department of Solid State Physics, Unversity of Debrecen, P.O. Box 2, H-4010 Debrecen, Hungary
}

(Received 25 September 2014; revised manuscript received 19 November 2014; published 4 December 2014)

\begin{abstract}
The jerky character of austenite-martensite phase transformation in $\mathrm{Ni}_{2} \mathrm{MnGa}$ single crystals (with $10 \mathrm{M}$ martensite structure) has been investigated by thermal cycling using a differential scanning calorimeter (DSC) and by detection of acoustic emissions (AEs) at low cooling and heating rates $(0.1 \mathrm{~K} / \mathrm{min}$ and below). It is illustrated that, besides the low cooling and heating rate, mass and surface roughness are also important parameters in optimizing the best signal/noise ratio in order to obtain individual peaks suitable for statistical analysis. Three types of samples, differing in the twin structure and twin boundary behavior, were investigated with and without surface roughening made by electro-erosion. The statistical analysis, carried out for both (thermal and acoustic) types of signals, provided power-law behavior. In calorimetric measurements the energy exponents, obtained in cooling, were the same within the experimental errors $(\varepsilon=1.7 \pm 0.2)$ for the three samples investigated. In acoustic emission experiments the energy and amplitude, $\alpha$, exponents were determined both for cooling and heating. The exponents for cooling and heating runs are slightly different. They are larger for heating for both $\alpha$ and $\varepsilon$, in accordance with the asymmetric acoustic activity: we observed higher acoustic activity (higher number of hits) during cooling. The effect of the surface roughness is negligible in the exponents (but higher acoustic activity corresponds to higher roughness) and the following values were obtained: $\varepsilon=1.5 \pm 0.1$ and $\alpha=2.1 \pm 0.1$ for cooling as well as $\varepsilon=1.8 \pm 0.1$ and $\alpha=2.6 \pm 0.1$ for heating. Our results are in accordance with the results of Gallardo et al. [Phys. Rev. B 81, 174102 (2010)] obtained in Cu based alloys: the exponents of the energy distributions, for both DSC and AE signals, were the same within the experimental errors. Furthermore, our exponents obtained from the AE measurements are close to the values obtained by Ludwig et al. $(\alpha=2.6 \pm 0.1$ and $\varepsilon=1.75 \pm 0.1$ ) [App. Phys. Lett. 94121901 (2009)] and Niemann et al. ( $\varepsilon=1.9 \pm 0.1)$ [Phys. Rev. B 86, 214101 (2012)] in $\mathrm{Ni}_{2} \mathrm{MnGa}$ alloys with similar $10 \mathrm{M}$ martensite structure.
\end{abstract}

DOI: 10.1103/PhysRevB.90.224103

PACS number(s): 81.30.Kf, 64.60.av, 43.90.+v, 65.40.-b

\section{INTRODUCTION}

It is well known that martensitic transformations are a stop-and-go type discontinuous processes, leading to a series of thermal or acoustic emission signals. Indeed, during the jerky propagation of interfaces avalanches are created and their individual peaks can be separated if the cooling rate is slow enough and then a statistical analysis of them is possible [1-6].

In calorimetric analysis of first-order phase transitions, due to the relatively fast scanning rate, usually an integrated peak of latent heat appears, which hides the fine structure of the jerky character of transformation. The fine structure can only be observed under much lower heating rates, and such types of investigations are rare in the literature [1,6-8]. Nevertheless if the scanning rate was slow enough (on the order of $0.1 \mathrm{~K} / \mathrm{h}$ ) the separation of the smooth base line from the individual spikes was successful in a $\mathrm{Cu}$ based $\left(\mathrm{Cu}_{67.64} \mathrm{Zn}_{16.71} \mathrm{Al}_{15.65}\right)$ alloy $[1,7,8]$. Statistical analysis of events showed that energy distribution followed a power law [1],

$$
P(E) \cong E^{-\varepsilon} \exp \left(-E / E_{c}\right)
$$

$[P(E)$ is the probability density, $\varepsilon$ is the critical exponent, and $E_{c}$ is the cutoff value], indicating a behavior near selforganized criticality.

Regarding the acoustic emission (AE) measurements, although they are also scarce [5], more experimental data

\footnotetext{
*Also at Department of Physics and Production Engineering, College of Nyíregyháza, Hungary.
}

are available and a number of studies have been published especially in Cu-based alloys. As reviewed in Ref. [5], results obtained in shape memory alloys confirmed that the austenite to martensite transformation shows avalanche criticality, and the characteristic exponents depend on the change of symmetry during the transition through variant multiplicity and on the driving mechanism. For example the amplitude and energy exponents, $\alpha$ as well as $\varepsilon$, decrease as the martensite symmetry changes from monoclinic to tetragonal (from $\alpha=3.0$ to 2.0, having an intermediate value 2.4 for orthorombic martensite, as well as from $\varepsilon=2.0$ to 1.6 , respectively). The effect of the driving mechanism is manifested in higher values for soft driving (when the externally applied field is controlled) as compared to hard driving [when the corresponding thermodynamic conjugated variable (generalized displacement) is controlled] [9]. It was concluded that, although during stress-driven (soft-driven) and thermally induced transition the system arrives at a single variant as well as at a multivariant structure, respectively, microscopic avalanche dynamics were similar for both of these unconstrained cases.

Furthermore, it was argued [5] that in hard-driven (straindriven) systems the critical exponents were independent of the number of cycles, while in soft cases the AE emission activity evolved from cycle to cycle, which was manifested in the change of critical exponents especially during the first cycles, accompanied also by a reduction of the dissipated energy. Regarding $\mathrm{Ni}_{2} \mathrm{MnGa}$ ferromagnetic alloys (with $10 \mathrm{M}$ structure) AE measurements of thermally induced transformation were investigated as a function of the external magnetic field [2] as well compressive stress [3] and it was obtained that 

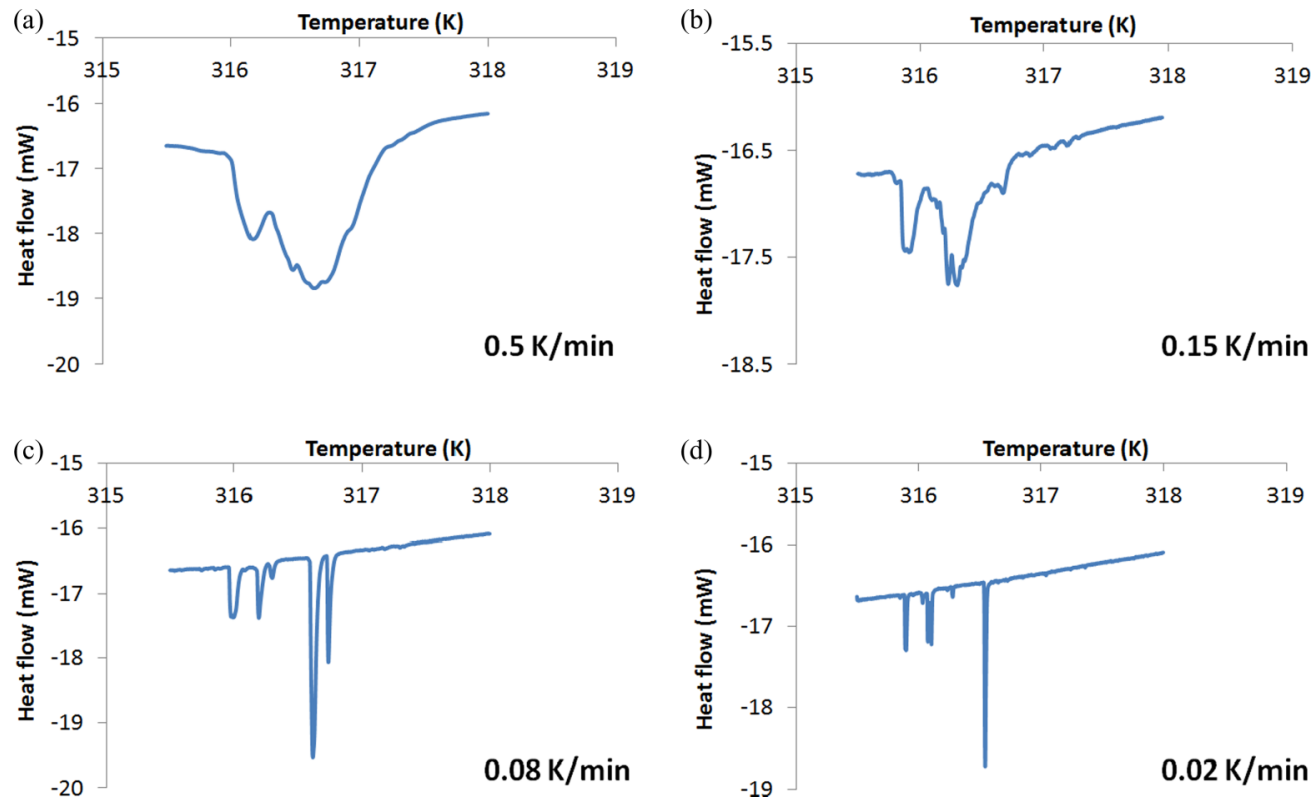

FIG. 1. (Color online) Effect of cooling rate on the shape of DSC curves for the shot-peened sample $(m=59 \mathrm{mg})$.

variant multiplicity (which was well controlled by the different levels of the constant external fields applied) has influence on the critical exponents. $\alpha$ and $\varepsilon$ showed about $25 \%$ decrease with increasing field. At the same time the authors of Refs. $[2,3]$ did not mention any effect of thermal cycling on critical exponents.

It is important to mention that till now there have only been two papers published on a $\mathrm{Cu}$-based shape memory alloy $[1,8]$ about the comparison of energy exponents obtained from $\mathrm{AE}$ and calorimetric measurements indicating that they were the same within the experimental errors.

In the last two decades a lot of work has been devoted to the so-called magnetically induced reorientation of twin variants in the martensitic state of $\mathrm{Ni}_{2} \mathrm{MnGa}$ shape memory alloys (see, e.g., Ref. [10]). This twinning-detwinning effect appears in a relatively modest magnetic field and can have many applications $[11,12]$. From the point of view of applications it is very important to increase twin boundary mobility. It has been obtained that in $\mathrm{Ni}_{2} \mathrm{MnGa}$ modulated martensite there are two kinds of mobile twin boundaries (called Type I and II) [13], of which only Type II shows very high mobility, and the temperature dependences of mobilities of the above mentioned two types of twin boundaries are also different [14]. Thus, it is an interesting question whether the thermal and AE noises and their kinetic exponents depend on the type of twin boundaries or not.

Accordingly in this paper simultaneous investigation of the jerky character of austenite-martensite phase transformation in $\mathrm{Ni}_{2} \mathrm{MnGa}$ single crystal by thermal cycling using a differential scanning calorimeter (DSC) and by an acoustic emission diagnostic device at low cooling rates (between 0.1 and $0.01 \mathrm{~K} / \mathrm{min}$ ) was carried out. Three types of single crystals, 50 at.\% $\mathrm{Ni}, 28.5$ at.\% $\mathrm{Mn}$, and 21.5 at.\% Ga (with $10 \mathrm{M}$ martensite structure), with different twin microstructures, were also compared.

\section{EXPERIMENT}

The three types of single crystalline Ni2MnGa billets $(1 \mathrm{~mm}$ $\times 2.5 \mathrm{~mm} \times 20 \mathrm{~mm}$ ) with $10 \mathrm{M}$ martensite structure were purchased from Adaptamat Co. (Finland). Adaptamat invented a special treatment (by application of combined effects of bending and external magnetic field [15]) to develop samples with very low twinning stress (about $0.2 \mathrm{MPa}$ ), containing so called Type II twin boundaries. These, in contrast to Type I twin boundaries oriented exactly parallel with the (101) axis (the twinning stress is about $1 \mathrm{MPa}$ ), have deviations by several degrees from (101) and also from the sample surface, since our samples were cut in such a way that faces were parallel with the $\{100\}$ austenite crystallographic planes. In summary, the samples provided by Adaptamat can be characterized as follows:

(a) Not treated: no special treatment, the surface is electropolished, twinning stress is $0.5-0.7 \mathrm{MPa}$, and elongation is larger than for surface treated samples. Unstable behavior and low fatigue.

(b) Shot peened: similar to (a) but stabilized by shot peening, good fatigue, and reproducible behavior under cycling. Twinning stress is about $1 \mathrm{MPa}$.

(c) Twin two: twinning stress is about $0.2-0.3 \mathrm{MPa}$, contains Type II twin boundaries.

Since surface defects and irregularities of the samples are nucleation centers, it is expected that the number of peaks in the thermal emission signals measured by the calorimeter will be higher if surface roughness is increased. Thus, in addition to asreceived samples, we also investigated samples after additional surface roughening (made by electro-erosion). The mass of single crystalline samples (i.e., the surface/volume ratio), cut from the original billets, is also an important parameter which can influence the number of thermal spikes. As a first trial we carried out DSC runs with samples of different masses (ranging between 9 and $108 \mathrm{mg}$ ) and it was observed that the 


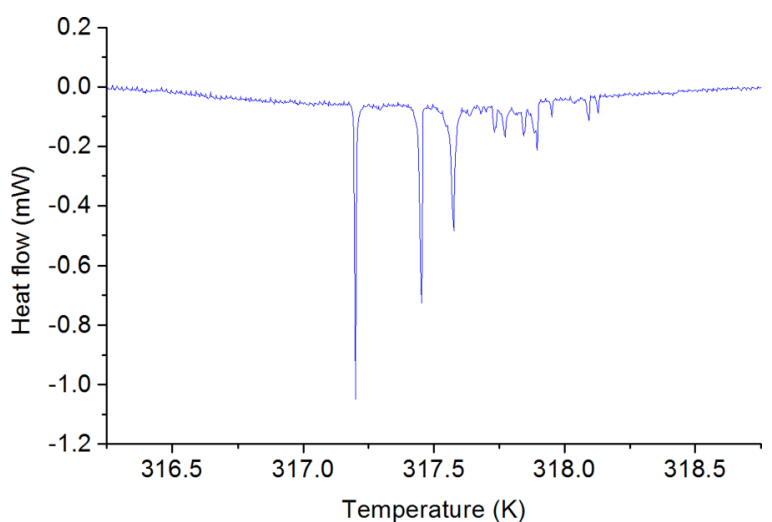

FIG. 2. (Color online) DSC results for cooling: heat flow (mW) versus temperature on the surface-roughened not-treated sample (cooling rate $0.02 \mathrm{~K} / \mathrm{min}, m=36 \mathrm{mg}$ ).

best signal/noise ratio was achieved if the mass was about 40 $\mathrm{mg}$. Thus the samples investigated further had dimensions of $1 \mathrm{~mm} \times 2.5 \mathrm{~mm} \times 2 \mathrm{~mm}$.

\section{A. Calorimetric measurements}

The obtained DSC curves show split peaks (jerky steps) related to the formation and growth of martensite nuclei if the mass of the sample is small and the rates of change of temperature is slow enough (Fig. 1). It can be seen that, for the shot-peened sample, by decreasing the cooling rate from $0.5 \mathrm{~K} / \mathrm{min}$ by about one order of magnitude, nice separate peaks can be obtained. Also note that well separated individual signals, similar to the ones shown in Figs. 1(c) and 1(d), were obtained only in the above type of sample and only during cooling. It was expected that by increasing the surface roughness, one could obtain more thermal emission signals in the calorimeter. Indeed, after additional surface roughening made by electro-erosion we obtained well split DSC peaks already for all the three types of samples for cooling, as illustrated for the not-treated sample in Fig. 2. Unfortunately, even after the surface roughening, we were not able to separate individual peaks during heating (see Fig. 3 for an illustration).

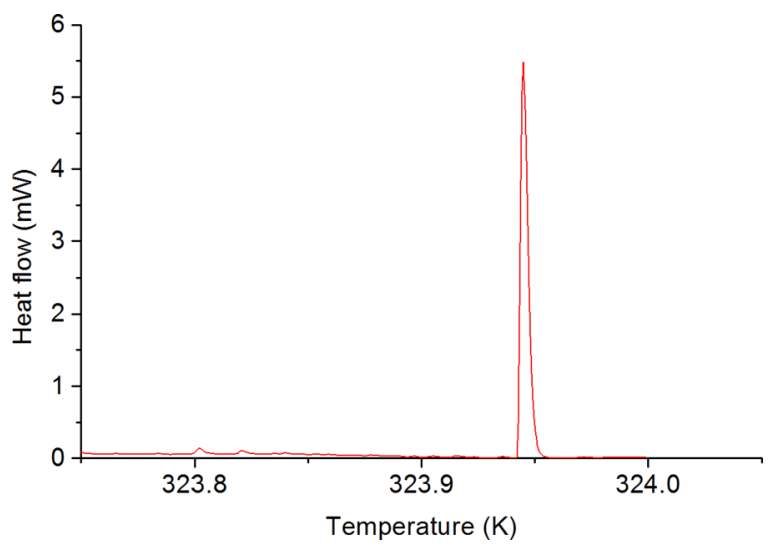

FIG. 3. (Color online) DSC curve during heating on the same sample shown in Fig. 2 (heating rate: $0.02 \mathrm{~K} / \mathrm{min}$ ).

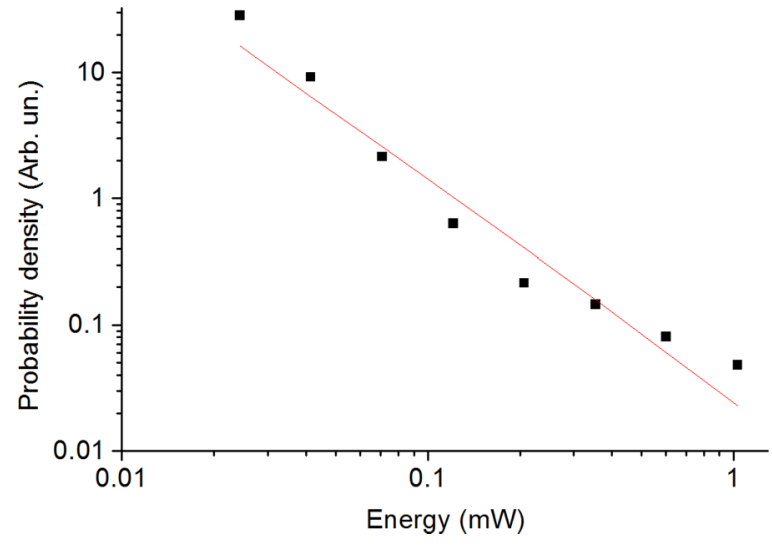

FIG. 4. (Color online) $\log P$ versus $\log E$ for cooling (summarized over 40 cycles) for the surface-roughened not-treated sample.

Statistical analysis of the obtained DSC signals was carried out similarly to the analyses in Refs. [1,4]. First, the main time constant of the experimental device had to be determined and filtered from the original signal. The time constant was determined in the following way: the sample was replaced by a calibration sample of similar dimensions and weight, and was heated by a sort laser pulse. From its response function the constant was determined $(\cong 6 \mathrm{~s})$. Before statistical analysis, distortions caused by the limitation of the device and background noise were filtered with a properly chosen threshold.

Energy distribution functions clearly showed power-law behavior, according to Eq. (1). The exponents characterizing the probability distributions $(\varepsilon)$ have been obtained using linear fitting to the $\log P$ versus $\log E$ functions. Figure 4 shows the probability distribution function of energies for cooling, summarized over 40 cycles for the surface-roughened nottreated sample. Table I shows energy exponents determined similarly for all samples investigated. The exponents were independent of the number of thermal cycles in all cases and their average is $1.7 \pm 0.2$.

\section{B. Acoustic emission measurements}

Sensophone AED 404 Acoustic Emission Diagnostic equipment (Geréb and Co. Ltd., Hungary) was used with piezoelectric sensors. The analog-to-digital converter sampling rate was $16 \mathrm{MHz}$ and the setup has a band-pass of $30 \mathrm{kHz}$ to $1 \mathrm{MHz}$. A $30 \mathrm{~dB}$ preamplifier and a maximum $100 \mathrm{~dB}$ main amplifier were used. Wave packets were recorded (Fig. 5) and the statistics of the distribution of amplitude and energy were

TABLE I. Energy exponents obtained from DSC measurements during cooling.

\begin{tabular}{lcc}
\hline \hline Cooling & Original & $\begin{array}{c}\text { Surface roughened by } \\
\text { electro-erosion }\end{array}$ \\
\hline Not treated & $\varepsilon$ & $\varepsilon$ \\
Twin two & & $1.7 \pm 0.2$ \\
Shot peened & $1.8 \pm 0.2$ & $1.5 \pm 0.2$ \\
\hline \hline
\end{tabular}




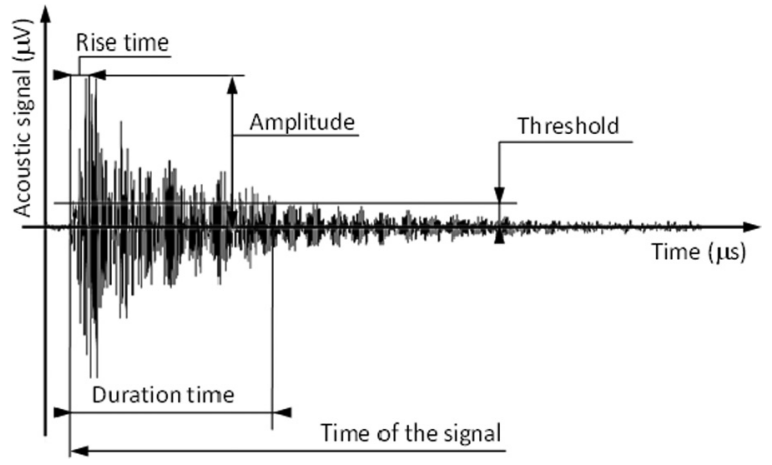

FIG. 5. Typical acoustic signal and measured parameters.

evaluated. The evaluation method was similar to the one used in Ref. [2]. Since the heating and cooling were done in the DSC, it was possible to measure thermal signals simultaneously with acoustic emission.

Statistical analysis of the acoustic signals (see also Fig. 6) showed that the amplitude and energy distributions satisfy power-law behavior (Fig. 7 and 8). As seen in Fig. 6, we observed asymmetric activity, i.e., numbers of hits during cooling and heating were different: they were much larger for cooling. Furthermore, it can also be seen that the main jumps on the curve of integral of acoustic events coincide well with the time window where the DSC activity was observed.

Tables II and III contain the exponents of energy and amplitude distributions ( $\alpha$ and $\varepsilon$ respectively) for all investigated samples at low cooling rates $(d T / d t \leqslant 0.1 \mathrm{~K} / \mathrm{min})$. For the calculation of exponents, the maximum likelihood method [16] is recommended as a more reliable estimation since it is independent of the data binning [1,3]. We checked all the exponents and their error bars with this method and the agreement was very good with the values obtained from the linear fitting of $\log P$ versus $\log E$ (or $\log A$ ) functions. Figure 9 shows, as an illustration, the power-law exponent as a function of $A_{\min }$ ( $A_{\min }$ is the lower cutoff of the amplitude) for

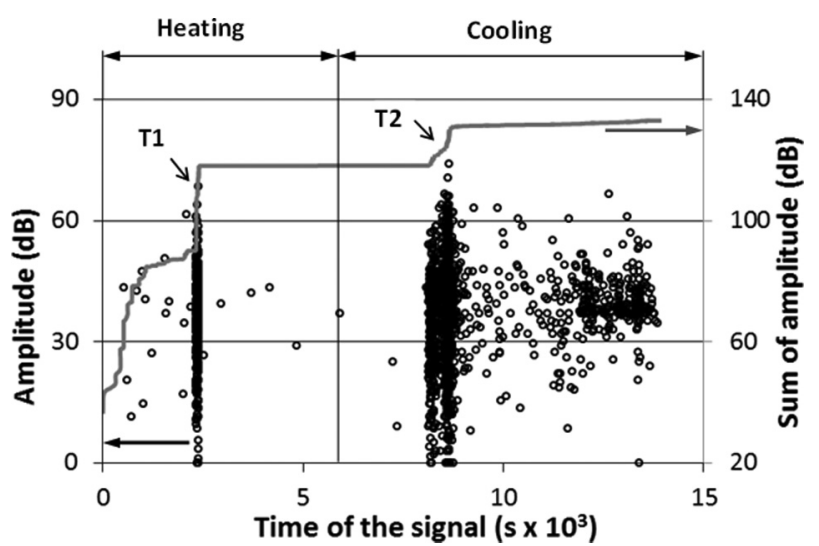

FIG. 6. Acoustic activity during heating and cooling $(0.1 \mathrm{~K} / \mathrm{min})$ in the shot-peened sample. Points indicate the amplitudes of hits (since, as indicated in Table II, a very large number of hits were observed, for the sake of clarity only every 15 th of them are presented). The upper continuous curve gives the sum or integral of amplitudes. Main jumps on this curve (T1 and T2) coincide well with the time window where the DSC activity was observed.

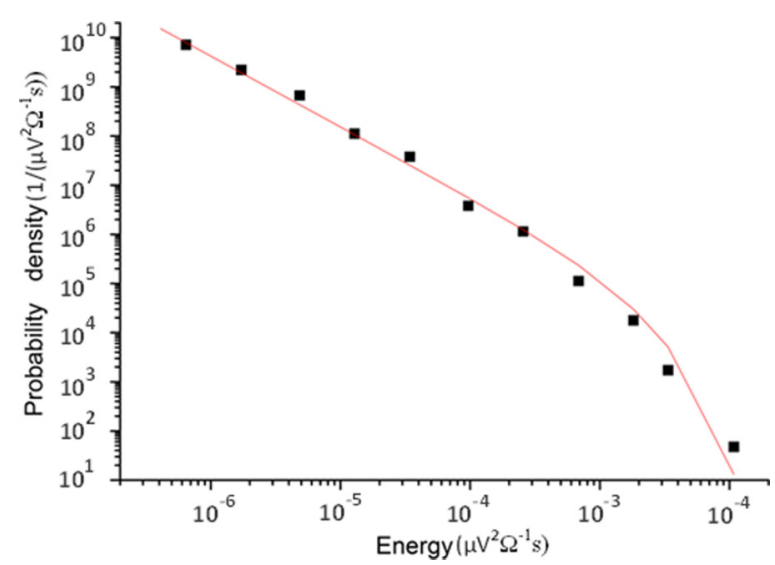

FIG. 7. (Color online) $\log P$ versus $\log E$, constructed from acoustic emission hits during cooling, for the surface-roughened shot-peened sample. Each point is calculated from large amount of hits (see also TABLE II). Note that the bins on the horizontal axis are logarithmic bins and the vertical axis is normalized by the linear bin width.

heating of the not-treated samples without surface roughening. Note that in Figs. 7 and 8 the points belonging to small values of $E$ and $A$, and corresponding to the first increasing part (before the plateau) of the $\varepsilon / \alpha$ versus $E_{\min } / A_{\min }$ functions, are not shown.

It can be seen that the values of $\varepsilon$ are similar and are about $1.5 \pm 0.1$ for cooling, and, except for the shot-peened sample, are about $1.8 \pm 0.1$ for heating. A similar tendency can be seen in Table III for parameters $\alpha$ : their average is about $2.0 \pm 0.1$ for cooling and a little bit larger for heating: $2.6 \pm 0.1$ (except again for the shot-peened sample, for which it is $2.1 \pm 0.1$ ).

It can be seen that the above exponents fulfill the required scale law [5] $(\alpha-1) /(\varepsilon-1)=2$, as well.

In order to investigate the effect of the number of cycles more precisely, we homogenized one not-treated sample at $1200 \mathrm{~K}$ for $48 \mathrm{~h}$ and water quenched it to room temperature. This was necessary because in our measurements the first 3-5 cycles were devoted to find the most optimal settlement for the temperature interval in the DSC, as well as to find the optimal

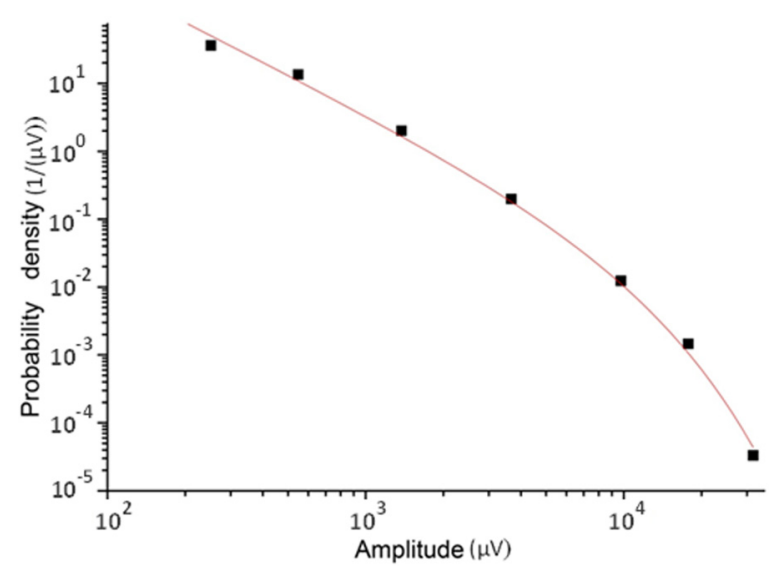

FIG. 8. (Color online) $\log P$ versus $\log A$, constructed from acoustic emission hits during cooling, for the surface-roughened shot-peened sample (see also the caption fod Fig. 7.). 
TABLE II. Energy exponents obtained from acoustic emission (the third and fifth columns show the number of hits detected)

\begin{tabular}{|c|c|c|c|c|}
\hline \multirow[b]{2}{*}{ Cooling } & \multicolumn{2}{|c|}{ Original } & \multicolumn{2}{|c|}{ Surface roughened } \\
\hline & $\varepsilon$ & Hits & $\varepsilon$ & Hits \\
\hline Not treated & $1.5 \pm 0.1$ & 18031 & $1.5 \pm 0.1$ & 25220 \\
\hline Twin two & $1.5 \pm 0.1$ & 19270 & $1.7 \pm 0.1$ & 25714 \\
\hline \multirow[t]{2}{*}{ Shot peened } & $1.5 \pm 0.1$ & 11848 & $1.5 \pm 0.1$ & 24577 \\
\hline & \multicolumn{2}{|c|}{ Original } & \multicolumn{2}{|c|}{ Surface roughened } \\
\hline Heating & $\varepsilon$ & Hits & $\varepsilon$ & Hits \\
\hline Not treated & $1.9 \pm 0.1$ & 7166 & $1.8 \pm 0.1$ & 21274 \\
\hline Twin two & $1.8 \pm 0.1$ & 9769 & $1.7 \pm 0.1$ & 27783 \\
\hline Shot peened & $1.6 \pm 0.1$ & 3205 & $1.5 \pm 0.1$ & 25400 \\
\hline
\end{tabular}

cooling rate. Figure 10 shows the energy exponents of the $\mathrm{AE}$ as a function of the number of cycles, $N$, up to $N=5$, together with the horizontal line giving the average value obtained by averaging over a high number of cycles. The results suggest that from the first to fifth heating cycles the energy exponents slightly increase, although the amount of the statistic error can obscure the week increase of exponents.

\section{DISCUSSION AND CONCLUSIONS}

We have shown that by changing the cooling rate, mass, and surface roughness of samples investigated in the DSC, well split peaks can be obtained during a thermally induced austenite-martensite transformation. These were suitable for statistical noise analysis at least for cooling.

The exponents of amplitude and energy distributions fulfill the well known scale law [5] $(\alpha-1) /(\varepsilon-1)=2$. The effect of additional surface roughness is negligible in the exponents (but higher noise activity corresponds to higher roughness).

Furthermore, we can conclude that energy exponents obtained from simultaneous measurements of thermal and acoustic signals from single crystalline $\mathrm{Ni}_{2} \mathrm{MnGa}$ samples are the same (DSC: $\varepsilon=1.7 \pm 0.2$ for cooling; AE: $\varepsilon=$ $1.5 \pm 0.1$ for cooling and $\varepsilon=1.8 \pm 0.1$ for heating) within the experimental errors, confirming the results of Ref. [1], where the same conclusion was drawn from similar experiments in polycrystalline $\mathrm{Cu}_{67.64} \mathrm{Zn}_{16.71} \mathrm{Al}_{15.65}$ samples.

Energy exponents did not show definite change with the number of cycles, although for a soft-driven case (to

TABLE III. Amplitude exponents obtained from acoustic emission.

\begin{tabular}{lcc}
\hline \hline Cooling & Original & $\begin{array}{c}\text { Surface roughened } \\
\alpha\end{array}$ \\
\hline Not treated & $\alpha$ & $2.03 \pm 0.04$ \\
Twin two & $2.01 \pm 0.04$ & $2.37 \pm 0.04$ \\
Shot peened & $1.97 \pm 0.04$ & $1.91 \pm 0.04$ \\
& Original & Surface roughened \\
Heating & $\alpha$ & $\alpha$ \\
\hline Not treated & $2.85 \pm 0.04$ & $2.70 \pm 0.04$ \\
Twin two & $2.57 \pm 0.04$ & $2.33 \pm 0.04$ \\
Shot peened & $2.08 \pm 0.04$ & $2.04 \pm 0.04$ \\
\hline \hline
\end{tabular}

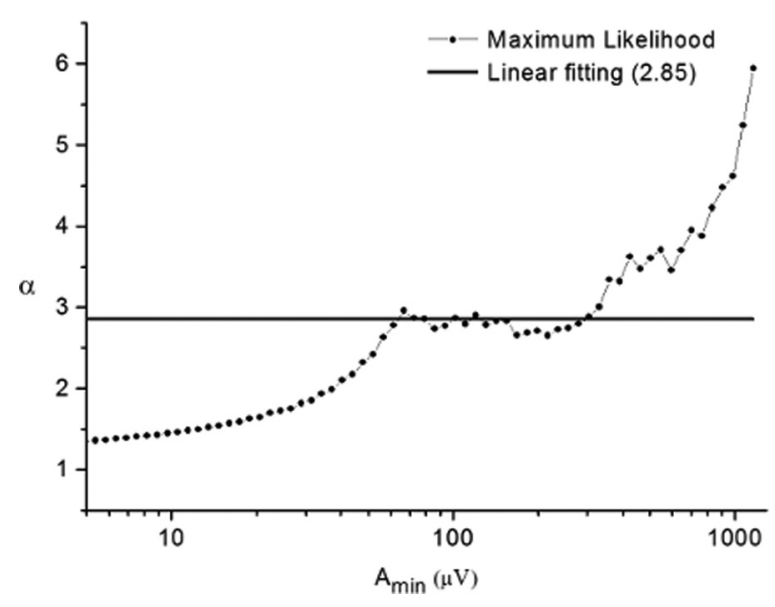

FIG. 9. Power-law exponent, $\alpha$, as a function of $A_{\min }$, using the maximum likelihood method. The horizontal line indicates the value obtained from a linear fitting of the $\log P$ versus $\log A$ function.

which, most probably, the thermally induced transitions belong [5]) such a change would be expected in the first cycles. As mentioned above, the slight increase was within the measurement errors in Fig. 10. Thus, this result should be checked later on in repeated measurements.

The exponents for the cooling and heating runs are slightly different: they are larger for heating for both $\alpha$ and $\varepsilon$. This is most probably related to the asymmetric acoustic activity: as mentioned (see Fig. 6), the number of hits was considerably larger for cooling than for heating. It is worth mentioning that a similar change in AE activity was observed in Ref. [17], and the relatively high activity during cooling was attributed to the relaxation of the martensite structure by twinning. The fact that a similar but a bit more moderate effect was observed for the thermal noise can be additionally related to nucleation problems: generally the nucleation of the low symmetry phase is more difficult than that of the high symmetry phase.

Our results can also be compared with acoustic emission measurements of Ludwig et al. [2], as well as Niemann et al. [3] where $\alpha=2.6$ and $\varepsilon=1.75$ [2] as well as $\varepsilon=1.9 \pm 0.1$ [3] were obtained at zero external field in $\mathrm{Ni}_{52} \mathrm{Mn}_{23} \mathrm{Ga}_{25}$. It can be concluded that the agreement is quite good. It is important to mention that the expected value for a cubic-monoclinic transformation (which is the case here) is 3 [5]. Indeed, it was

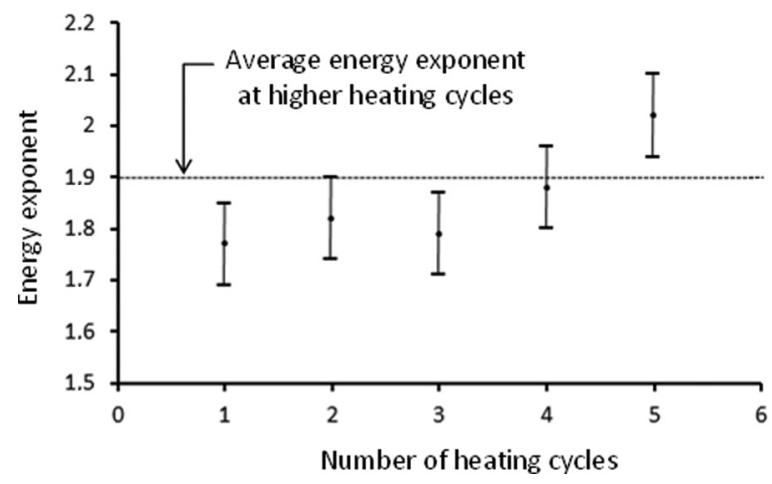

FIG. 10. Dependence of energy exponents of the not-treated sample on the number of heating cycles. 
mentioned in Ref. [5] that the value of 2.6, which was closer to 2.4 (characteristic for a cubic-orthorhombic transition) is surprising, and the origin of it was not understood. The authors of Ref. [5] argued that this could be related to the adaptive nature of the martensite structure. Indeed, in Ref. [3] it was pointed out that the $10 \mathrm{M}$ martensite structure has a monoclinic symmetry, which allows a multiplicity of 12 [18], and the value $\varepsilon=1.9 \pm 0.1$ agree well with the $\varepsilon=2.0 \pm 0.2$ expected for monoclinic martensites [5]. On the other hand the value of $\alpha \cong 2.6$ obtained in Ref. [2] and by us for heating (and $\alpha=2.1$ for cooling) is less than the expected value 3 for monoclinic martensite. Thus, at the moment it is difficult to decide whether our results fit the monoclinic or the orthorhombic transformation. According to Table I in Ref. [5] for orthorombic structure the expected values are $\alpha \cong 2.4 \pm 0.1$ as well as a value intermediate between $\varepsilon=2.0 \pm 0.1$ and $1.6 \pm 0.1$. Thus the agreement with the orthorombic transformation is also not bad. Indeed, it was concluded in Ref. [5] that these exponents were close to those expected for this type of transition, and the authors expressed their opinion that it is surprising and not well understood yet. The adaptive nature of the martensite in
$\mathrm{Ni}_{2} \mathrm{MnGa}$ alloys [19] was offered as a possible explanation for this discrepancy [5]. Further investigations are desired to clear up the situation. This is also supported by the following result. The preliminary treatment (intended to influence the twin mobility in the martensite state) has the following effect: the exponents are a bit smaller for shot-peened samples. As mentioned in the introduction, this sample had stabilized fine twin microstructure, and average values of energy and amplitude exponents from $\mathrm{AE}$ measurements are about $\varepsilon=$ 1.5 and $\alpha=2.0$ and; these are close to those expected for tetragonal martensite [5]: $\varepsilon=1.6$ and $\alpha=2.0$.

\section{ACKNOWLEDGMENTS}

This work is supported by the TÁMOP-4.2.2.A11/1/KONV-2012-0036 and TÁMOP-4.2.2/B-10/1-20100024 projects, which are implemented through the New Hungary Development Plan, co-financed by the European Social Fund and the European Regional Development Fund; and by OTKA Board of Hungary (K84065). The authors are indebted to Dr. Zoltán Halász for discussions on the statistical analysis.
[1] M. C. Gallardo, J. Manchado, F. J. Romero, J. del Cerro, E. K. H. Salje, A. Planes, E. Vives, R. Romero, and M. Stipcich, Phys. Rev. B 81, 174102 (2010).

[2] B. Ludwig, C. Strothkaemper, U. Klemradt, X. Moya, L. Mañosa, E. Vives, and A. Planes, App. Phys. Lett. 94121901 (2009).

[3] R. Niemann, J. Baró, O. Heczko, L. Schultz, S. Fähler, E. Vives, L. Mañosa, and A. Planes, Phys. Rev. B 86, 214101 (2012)

[4] L. Carrillo and J. Ortín, Phys. Rev. B 56, 11508 (1997).

[5] A. Planes, L. Mañosa, and E. Vives, J. Alloys Compounds 577 (Suppl. 1), S699 (2013).

[6] E. K. H. Salje, Solid State Phenom. 172-174, 3 (2011).

[7] F. J. Romero, J. Manchado, J. M. Martín-Olalla, M. C. Gallardo, and E. K. H. Salje, Appl. Phys. Lett. 99, 011906 (2011).

[8] J. Baró, J.-M. Martín-Olalla, F. J. Romero, M. C. Gallardo, E. K. H. Salje, E. Vives, and A. Planes, J. Phys. Condens. Matter 26, 125401 (2014).

[9] E. Vives, D. Soto-Parra, L. Mañosa, R. Romero, and A. Planes, Phys. Rev. B 80, 180101 (2009).
[10] O. Heczko, A. Sozinov, and K. Ullakko, IEEE Trans. Magn. 36, 3266 (2000).

[11] K. Ullakko, L. Wendell, A. Smith, P. Müllner, and G. Hampikian, Smart Mater. Struct. 21, 115020 (2012).

[12] L. Straka and O. Heczko, IEEE Trans. Magn. 39, 3402 (2003).

[13] L. Straka, O. Heczko, H. Seiner, N. Lanska, J. Drahokoupil, A. Soroka, S. Fähler, H. Hänninen, and A. Sozinov, Acta Mater. 59, 7450 (2011).

[14] O. Heczko, V. Kopecký, A. Sozinov, and L. Straka, Appl. Phys. Lett. 103, 072405 (2013).

[15] L. Straka, H. Hänninen, A. Soroka, and A. Sozinov, J. Phys.: Conf. Ser. 303, 012079 (2011).

[16] A. Clauset, C. R. Shalizi, and M. E. Newman, SIAM Rev. 51, 661 (2009).

[17] L. Straka, V. Novák, M. Landa, and O. Heczko, Mater. Sci. Eng. A 374, 263 (2004).

[18] K. Bhattacharya, Microstructure of Martensite (Oxford University Press, Oxford, 2007).

[19] S. Kaufmann, U. K. Rößler, O. Heczko, M. Wuttig, J. Buschbeck, L. Schultz, and S. Fähler, Phys. Rev. Lett. 104, 145702 (2010). 